\title{
A new "ASAP Scoring System and Risk Table" in patients with Atypical Small Acinar Proliferation (ASAP) to predict the second prostate biopsy outcomes
}

\author{
Caner Ediz ${ }^{1}$, Serkan Akan², Neslihan Kaya Terzi ${ }^{1}$, and Aysenur Ihvan ${ }^{3}$ \\ ${ }^{1}$ Sultan Abdulhamid Han Egitim ve Arastirma Hastanesi \\ ${ }^{2}$ Sultan Abdülhamid Han Training and Research Hospital \\ ${ }^{3}$ Umraniye Training and Research Hospital
}

June 8, 2021

\begin{abstract}
Background: To discuss the necessity of the second prostate biopsy in the patients with atypical small acinar proliferation (ASAP) and to develop a scoring system and risk table as a new re-biopsy criteria. Methods: 2845 patients who were performed transrectal ultrasonography-guided prostate biopsy between January 2008 and May 2019 were evaluated. 128 patients, whose data were reached, were enrolled into the study. Before the first and the second biopsy, tPSA, fPSA, f/tPSA rate and PSADensity assessment and changes in these parameters between the two biopsies were recorded. "ASAP Scoring System and risk table" (ASS-RT) was evaluated before the second biopsy. Results: The mean age of 128 patients with ASAP was $62.9 \pm 7.8$ years. The ASS-RT scores of the patients with PCa were statistically significantly higher than the patients with non-PCa (p: 0.001). In the ROC curve analysis of ASS-RT, area under the curve was 0.804 and the standard error was 0.04 . The area under the ROC curve was significantly higher than 0.5 (p:0.001). The cut-off point of ASS-RT score in diagnosis of malignancy was [?] 7. The sensitivity of this value was found to be $60.8 \%$ and its specificity as $80.5 \%$. Conclusions: The threshold value for the ASS-RT score may be used as 7 and the second biopsy may be performed immediately to patients over this value. We think that there may be no need for a second biopsy if the ASS-RT score under the 7 (especially low-risk group) before the second biopsy.
\end{abstract}

\section{Introduction}

Prostate cancer (PCa) is the most diagnosed cancer, apart from skin cancer, on the men over 70 years of age $^{1}$. Needle core prostate biopsy is a gold standart to diagnose of PCa. Atypical small acinar proliferation (ASAP) first defined by Bostwick et al. ${ }^{2}$ and described as a focus of small acinar structures $(<0.4 \mathrm{~mm})$ formed by atypical epithelial cells in needle core prostate biopsy. ASAP is diagnosed at $5 \%$ of all prostate biopsies ${ }^{3,4}$ and considered precancerous lesion of prostate tissues ${ }^{5,6}$. Second prostate biopsy is routinely recommended by current National Comprehensive Cancer Network (NCCN) and European Association of Urology (EAU) guidelines ${ }^{7,8}$ due to $30-40 \%$ PCa risk for the patients reported ASAP as a result of the initial biopsy $^{9,10}$.

Morbidity associated with needle core prostate biopsy is a problem to both patients and clinicians ${ }^{11-13}$. Some of the patients want to delay the second biopsy as much as possible. Furthermore, TRUS-guided prostate biopsy is high cost process ${ }^{14}$. In addition to its morbidity and high cost, it is also not associated clinically significant or high risk $\mathrm{PCa}^{15}$. Therefore, the second prostate biopsy has been controversial for many years.

In this study, we aimed to discuss the requirement of the repeat biopsy routinely for the patients with ASAP and which patients should be absolutely evaluated. We offered a scoring system and risk table that may be 
able to guide the decision of second prostate biopsy.

\section{Materials and Methods}

This retrospective study was approved by the local Ethics Committee and conducted according to the principles of World Medical Association Declaration of Helsinki 'Ethical Principles for Medical Research Involving Human Subjects.

\section{Patient Population}

2845 cases were performed transrectal ultrasonography (TRUS) guided prostate biopsy due to elevated PSA level and/or significant dijital rectal examination (DRE) findings in our clinic between January 2008 and May 2019 were evaluated. In 238 of 2295 prostate biopsy patients ASAP was revealed and 128 cases whose data were reached taken into the study.

\section{Inclusion and Exclusion Criteria}

The criteria for inclusion in the study were as follows: patients with elevated prostate specific antigen (PSA) level and/or anormal findings in digital rectal examination. Patients with diagnosed a diesease of urinary tract infections, coagulopathies, patients who have had surgery in the past year or patients who received only 5-alfa reductase inhibitory treatment or as a combination therapy and patients with inadequate data were also excluded from the study.

\section{Study Design}

The patients' medical records were retrospectively reviewed. Patients age, prostate volume (PV) total PSA (tPSA) and fPSA level (fPSA), rate of percentage of free to total prostate specific antigen (f/tPSA), PSADensity (PSA-D) $(\mathrm{mL})$ level were evaluated. Also PSA velocity $\left(\mathrm{PSA}_{\mathrm{v}}\right)$ was calculated according to difference of PSA forms $\left(\mathrm{tPSA}_{\mathrm{v}}, \mathrm{fPSA}_{\mathrm{v}}, \mathrm{f} / \mathrm{tPSA}_{\mathrm{v}}\right.$ and PSA-D $\mathrm{D}_{\mathrm{v}}$ ) between first and second biopsy.

\section{ASAP Scoring System And Risk Table (ASS-RT)}

All the patients were divided into two groups according to second prostate biopsy pathology result. Group 1 was descripted as a patients with benign pathology (Prostatitis or Benign Prostate Hyperplasia) while group 2 was patients with prostate cancer (PCa) in second prostate biopsy. We designed a scoring system and risk table for evaluation of patients with ASAP before the repeat biopsy (Table 1).

Four benchmarks were scored from 0 to 3 or 4 point in the scoring system and risk table. tPSA was scored from 1 to 3 point according to $0-<4,4-10$ and $>10 \mathrm{ng} / \mathrm{dl}$. $\mathrm{f} / \mathrm{tPSA}$ was scored from 1 to 3 point according to $<0.15,0.15-0.25$ and $>0.25 \%$. PSA-D was scored from 1 to 4 point according to $<0.08,0.08-0.129,0.13-0.15$ and $>0.15 \mathrm{ng} / \mathrm{dl} / \mathrm{ml}$. PSA $A_{\mathrm{v}}$ was scored 0 or 1 point according to the decrease or increase of tPSA before second prostate biopsy. Total score of ASS-RT was from 3 to 11 point. Last, subsequent to all assessment, all patients were divided according to total score as low (3-5), intermediate (6-8) or high (9-11) risk.

\section{TRUS Guided Prostate Biopsy Procedure}

One day before the procodure, oral administraten of 500-mg levofloxacin and 400-mg etodolac was started and it was continued after the biopsy. The day of biopsy a rectal enema $(250 \mathrm{~mL})$ was performed before the biopsy. The biopsy was performed while the patient was in the left lateral position with the thighs flexed. The procedure was performed under the guidance of ultrasound device with a $7.5 \mathrm{mHz}$ biplanar probe.

The biopsy was performed on an outpatient basis in a room equipped with all material necessary for emergency intervention. Sedation and anesthesia were not achieved. 10 minutes before the procedure, periprostatic nerve blockade was performed in addition to perianal intrarectal lidocain gel. Injections were delivered at the angle between the seminal vesicle and prostate on each side using $5 \mathrm{cc}$ of $2 \%$ lidocain. The biopsies were performed by multiple experienced urologists. In initial biopsy, standard 12 (both lateral and medial biopsies from the base, medial and apex on the right and left side of the prostatic peripheral zone) or 10 core prostate 
biopsy was performed. Second prostate biopsy was performed in patients within a period of 3-6 months after the initial biopsy. The core number of taken at the second biopsy was 16 or 18 .

Statistical Analysis

Statistical analyses were performed using SPSS Statistics 22.0 software (SPSS Inc., Chicago, IL, USA) and Microsoft excel computer programs. The normality hypothesis was tested using the Shapiro-Wilk Test during data analysis. Descriptive levels are presented as means and standard deviations for normally distributed, and medians and minimum/maximum for other than normally distributed. According to normality test results, Student t and Mann Whitney U test were applied to compare first and second biopsy results. Receiver operating characteristic (ROC) curves were used to quantify the predictive accuracy of the results. Two-tailed levels of $\mathrm{P}<.05$ were considered statistically significant.

\section{Results}

The mean age of all the patients with ASAP was $62.9 \pm 7.8$ years (40-78). In first prostate biopsy, the mean tPSA level, fPSA level, f/tPSA rate and PSA-D level were found as $8.63 \mathrm{ng} / \mathrm{mL}(0.9-32.5), 1.59 \mathrm{ng} / \mathrm{mL}$ (0.0017-8.9), 0.19 (0.0014-0.79), and $0.19 \mathrm{ng} / \mathrm{mL} / \mathrm{cc}(0.01-0.98)$, respectively. The second prostate biopsy results were reported as benign prostate pathology for 77 patients $(60.2 \%)$ and PCa for 51 patients $(39.8 \%)$ (ISUP Grade Group 1: 36, ISUP Grade Group 2: 10 and ISUP Grade Group 4: 5 patients). The mean PV in group 1 and 2 were $58.96 \pm 30.66$ and $47.71 \pm 25.44 \mathrm{~mL}$, respectively. This difference was found as statistically significant $(\mathrm{p}<0.037)$.

According to the first biopsy result of patients with PCa, tPSA levels increased before the second prostate biopsy while the patients with benign prostate pathology decreased and this difference was found as statistically significant (p: 0.001). The increase in fPSA levels before the second prostate biopsy compared to the first biopsy result of the patients with PCa was statistically significantly lower than the cases with benign prostate pathology (p: 0.002). Also f/tPSA levels decreased in group 2 before the second prostate biopsy while it increased in patients with benign prostate pathology and this difference was found as statistically significant (p: 0.001). In group 2, PSA-D levels increased before the second prostate biopsy while it decreased in patients with benign prostate pathology and this difference was found as statistically significant (p: 0.001) (Table 2). Changes in "PSA forms" before the second prostate biopsy and statistical evaluation of these parameters between the two groups were summarized in Table 3. ASS-RT scores of the patients with PCa were statistically significantly higher than the patients with benign prostate pathology (p:0.001).

The ROC curve of ASS-RT score was evaluated in the diagnosis of PCa. The area under the curve was 0.804 and the standard error was 0.04 . The area under the ROC curve was significantly higher than 0.5 (p: 0.001; p $<0.05$ ). The cut-off point of the ASS-RT score in diagnosis of PCa was [?] 7. The sensitivity and specificity of threshold value were found as $60.8 \%$ and $80.5 \%$, respectively (Figure 1).

The ROC curve of $\mathrm{PSA}_{\mathrm{v}}$ was evaluated in the diagnosis of PCa. The area under the curve was 0.790 and standard error was 0.04. The area under the ROC curve was significantly higher than 0.5 (p: $0.001 ; \mathrm{p}<0.05$ ). The detirmened cut-off point of the tPSA $\mathrm{v}_{\mathrm{v}}$ in the diagnosis of PCa was $>0.4$. The sensitivity and specificity of threshold value were found as $88.2 \%$ and $71.4 \%$, respectively (Figure 1).

The ROC curve of $\mathrm{PPSA}_{\mathrm{v}}$ was evaluated in the diagnosis of PCa. The area under the curve was 0.664 and standard error was 0.05. The area under the ROC curve was significantly higher than 0.5 (p: $0.001 ; \mathrm{p}<0.05$ ). The detected cut-off point of the $\mathrm{PPSA}_{\mathrm{v}}$ in diagnosis of PCa was [?]0.12. The sensitivity and specificity of threshold value were found as $78.4 \%$ and $62.3 \%$, respectively (Figure 1).

The ROC curve of $\mathrm{f} / \mathrm{tPSA}_{\mathrm{v}}$ was evaluated in the diagnosis of PCa. The area under the curve was 0.696 and standard error was 0.05 . The area under the ROC curve was significantly higher than 0.5 (p: $0.001 ; \mathrm{p}$ $<0.05$ ). The detected cut-off point of $\mathrm{f} / \mathrm{tPSA}_{\mathrm{v}}$ in diagnosis of PCa was [?]0.02 The sensitivity and specificity of threshold value were found as $92.2 \%$ and $40.3 \%$, respectively (Figure 1).

The ROC curve of PSA- $\mathrm{D}_{\mathrm{v}}$ was evaluated in the diagnosis of PCa. The area under the curve was 0.745 
and standard error was 0.04 . The area under the ROC curve was significantly higher than 0.5 (p: $0.001 ; \mathrm{p}$ $<0.05$ ). The detected cut-off point of the PSA- $\mathrm{D}_{\mathrm{v}}$ in the diagnosis of PCa was $>0.02$. The sensitivity and specificity of threshold value were found as $88.2 \%$ and $58.4 \%$, respectively (Figure 1).

\section{Discussion}

Atypical small acinar proliferation is a pathology that should be re-evaluated with a second biopsy in cases where small foci cannot be clearly defined histopathologically and in cases where differential diagnosis of PCa cannot be definitively performed. Detection rate of patients with ASAP in our biopsy cohort was $11.28 \%$. This rate has increased especially in the past few years. We think that these rates have increased as a result of decreasing the tPSA threshold value from 4 to the $2.5 \mathrm{ng} / \mathrm{mL}$ in recent years. Our re-biopsy rate was $53.78 \%$. In the literature, there are second biopsy rates varying between $47-63 \%^{16}$ and it was compatible with the literature. The question that should be responsed is the reasons why the patients avoid from the second biopsy. It is difficult to convince patients who need a second biopsy due to psychological or physical difficulties of the biopsy procedure. This rate reflects the public's approach to prostate biopsy, especially in Muslim countries. Therefore, the necessity of a second prostate biopsy is always scrutinized. Considering that it causes additional cost and morbidity, this decision should be made carefully.

In our patients with ASAP, PCa was occured $39.84 \%$ of the second prostate biopsy. In the literature, the diagnosis rate of $\mathrm{PCa}$ in patients with ASAP in the second biopsy ranges from 39 to $42 \%^{17,18}$. The relationship between ASAP and PCa has been evaluated for many years. Currently, there is no published algorithm for patients with ASAP to avoid unnecessary biopsy, and a second prostate biopsy is routinely applied to all patients diagnosed with ASAP in line with the recommendations of the guidelines. There are many studies in the literature focused on the diagnosis of PCa as a result of the second biopsy of patients diagnosed with ASAP and the predictive values of the all PSA forms were evaluated to explain relationship between ASAP and PCa in these studies.

Several nomograms have been reported in the literature to increase detection rates of PCa in recurrent prostate biopsies. In a study by Sakura et al., a new repeat biopsy nomogram was developed, including patients' age, f/tPSA rate, PV, etc ${ }^{19}$. However, patients diagnosed with ASAP were not included in the study. Yanke et al. and Moussa et al. were reported a nomogram that contains PSA slopen and history of high grade intraepithelial neoplasm or ASAP ${ }^{20,21}$. A study by Corona et al. was reported a nomogram that the cumulative number of negative cores obtained, PSA slope, history of high grade prostatic intraepithelial neoplasia and history of ASAP were associated with repeat biopsy findings ${ }^{22}$. These studies created nomograms with many parameters but were not enough to be routinely recommended in the guidelines. In addition, evaluating some parameters meant an extra cost increase. For example to evaluate PSA slope at least 3 measurements are required. All the nomograms were not examined specifically for patients with ASAP. It also does not point the alteration of tPSA between two biopsies. In ASS-RT, we have defined, used 4 benchmarks that can be measured effectively in prostate biopsy decision. Another difference of ASS-RT from the others is that it divides the patients into risk groups according to the score they received. When the number of patients in both groups is compared, we think that the risk group classification is extremely important and statistically significant (Table 4). A study on the classification of patients with ASAP as low and high risk by risk group was published in 2005 by Scattoni et al. and they showed there is no difference of getting cancer between these two groups after repeated biopsies ${ }^{23}$. There was no difference between risk groups in terms of detection of PCa. Contrary to this study, the use of "ASAP Scoring System and risk table "increases the predictability of PCa diagnosis in our study. As a result of the second biopsy in our study, $8 \%$ of the patients with benign prostate pathology were in the high-risk group, while 50.98\% (26/51) of the patients with PCa were in the high-risk group. However, Gleason score of the most of the patients with $\mathrm{PCa}(70.58 \%)$ was $3+3$ and the rate of clinically significant PCa was found as $29.42 \%$ in group 2 . Unfortunately, the parameter that would provide sufficient distinction could not be obtained in the intermediate risk group.

Considering the relationship between ASAP and clinically insignificant PCa, too high tPSA levels are not expected in patients with ASAP. The mean tPSA level of the cohort in our study ranged from 4.0 to 10.0 
$\mathrm{ng} / \mathrm{ml}$, known as the gray zone. In a previous study, it was reported that the rate of PCa detection in the first biopsy was $25.1 \%$ and the rate of clinically significant PCa detection was lower in men with PSA levels of $4.0-10.0 \mathrm{ng} / \mathrm{ml}^{24}$. As mentioned before, this rate is approximately $40 \%$ in patients diagnosed with ASAP. However, our clinically significant PCa rate was $11.71 \%$ in all patients with ASAP. Therefore, in patients with ASAP, the rate of clinically significant PCa as a result of the second biopsy is less than feared and we think that the best way to prevent high costs with increased morbidity in re-biopsy is to better identify to high risk patients by using of ASS-RT.

The main limitation of the study was retrospective planning. Unfortunately, the number of patients with data loss prevented more patients in the cohort. In fact, another factor that is not a limitation but it may increase the effectiveness of the current ASS-RT is the time elapsed between two biopsies and this factor was not examined in detail in our study. The time between two biopsies is perhaps an effective factor in the formation of the final pathology in patients diagnosed with ASAP.

\section{Conclusions}

"ASAP Scoring System and risk table" may provide additional information to urologist for assessment of clinical options in patient with ASAP. Total PSA, fPSA, f/tPSA rate and PSA-D levels may be routinely evaluated in the patients with ASAP before the second biopsy (4-6 weeks after the first biopsy). Alterations in these parameters may be considered and accordingly, a re-biopsy decision may be made. The risk group classification of patients by using of ASS-RT may help to better define the patients who need to perform a second biopsy. Thus, early diagnosis possibility for high risk patients will be possible. It should be known that we also need more data to validate this scoring system and risk table.

\section{Author Contributions}

EC contributed to the conception and design of the study, EC and AS collection of data, drafting, EC, IA and $\mathrm{KN}$ revision the manuscript, in preparing tables and performing statistical analysis. Author read and approved the final manuscript.

Conflict of interest: There is no conflict of interest

\section{Acknowledgments}

The author would like to thank staff of the Department of Urology in Sultan Abdulhamid Han Education and Research Hospital.

REFERENCES 1. Siegel RL, Miller KD, Jemal A. Cancer statistics, 2018. CA Cancer J Clin. $2018 ; 68(1): 7-30$.

2. Bostwick DG, Qian J, Frankel K. The incidence of high grade prostatic intraepithelial neoplasia in needle biopsies. J Urol.1995;154(5):1791-1794.

3. Cheville JC, Reznicek MJ, Bostwick DG. The focus of "atypical glands, suspicious for malignancy" in prostatic needle biopsy specimens: incidence, histologic features, and clinical follow-up of cases diagnosed in a community practice. Am J Clin Pathol.1997;108(6):633-640.

4. Montironi R, Scattoni V, Mazzucchelli R, Lopez-Beltran A, Bostwick DG, Montorsi F. Atypical foci suspicious but not diagnostic of malignancy in prostate needle biopsies (also referred to as "atypical small acinar proliferation suspicious for but not diagnostic of malignancy"). Eur Urol. 2006;50(4):666-674.

5. Srirangam V, Rai BP, Abroaf A, et al. Atypical Small Acinar Proliferation and High Grade Prostatic Intraepithelial Neoplasia: Should We Be Concerned? An Observational Cohort Study with a Minimum Follow-Up of 3 Years. Curr Urol. 2017;10(4):199-205.

6. Leone L, Lacetera V, Montironi R, et al. Biopsy follow-up in patients with isolated atypical small acinar proliferation (ASAP) in prostate biopsy. Arch Ital Urol Androl. 2014;86(4):332-335. 
7. Carroll PR, Parsons JK, Andriole G, et al. Prostate cancer early detection, version 1.2014. Featured updates to the NCCN Guidelines.J Natl Compr Canc Netw. 2014;12(9):1211-1219; quiz 1219.

8. Mottet N, Bellmunt J, Bolla M, et al. EAU-ESTRO-SIOG Guidelines on Prostate Cancer. Part 1: Screening, Diagnosis, and Local Treatment with Curative Intent. Eur Urol. 2017;71(4):618-629.

9. Epstein JI, Herawi M. Prostate needle biopsies containing prostatic intraepithelial neoplasia or atypical foci suspicious for carcinoma: implications for patient care. J Urol. 2006;175(3 Pt 1):820-834.

10. Ericson KJ, Wenger HC, Rosen AM, et al. Prostate cancer detection following diagnosis of atypical small acinar proliferation. Can J Urol. 2017;24(2):8714-8720.

11. Loeb S, Carter HB, Berndt SI, Ricker W, Schaeffer EM. Is repeat prostate biopsy associated with a greater risk of hospitalization? Data from SEER-Medicare. J Urol. 2013;189(3):867-870.

12. Loeb S, Vellekoop A, Ahmed HU, et al. Systematic review of complications of prostate biopsy. Eur Urol. 2013;64(6):876-892.

13. Quaresima L, Lacetera V, Leone L, et al. The impact of repeated prostate biopsies on sexual function and urinary symptoms in patients with diagnosis of Atypical Small Acinar Proliferation (ASAP): can ecoDoppler reduce side effects? Arch Ital Urol Androl.2014;86(4):356-358.

14. Fandella A. Analysis of costs of transrectal prostate biopsy. Urologia. 2011;78(4):288-292.

15. Ynalvez LA, Kosarek CD, Kerr PS, et al. Atypical small acinar proliferation at index prostate biopsy: rethinking the re-biopsy paradigm. Int Urol Nephrol. 2018;50(1):1-6.

16. Leone A, Rotker K, Butler C, et al. Atypical Small Acinar Proliferation: Repeat Biopsy and Detection of High Grade Prostate Cancer. Prostate Cancer. 2015;2015:810159.

17. Leone A, Gershman B, Rotker K, et al. Atypical small acinar proliferation (ASAP): Is a repeat biopsy necessary ASAP? A multi-institutional review. Prostate Cancer Prostatic Dis.2016;19(1):68-71.

18. Mallen E, Gil P, Sancho C, et al. Atypical small acinar proliferation: review of a series of 64 patients. Scand J Urol Nephrol. 2006;40(4):272-275.

19. Sakura M, Kawakami S, Ishioka J, et al. A novel repeat biopsy nomogram based on three-dimensional extended biopsy. Urology.2011;77(4):915-920.

20. Moussa AS, Jones JS, Yu C, Fareed K, Kattan MW. Development and validation of a nomogram for predicting a positive repeat prostate biopsy in patients with a previous negative biopsy session in the era of extended prostate sampling. BJU Int. 2010;106(9):1309-1314.

21. Yanke BV, Gonen M, Scardino PT, Kattan MW. Validation of a nomogram for predicting positive repeat biopsy for prostate cancer. J Urol. 2005;173(2):421-424.

22. Lopez-Corona E, Ohori M, Scardino PT, Reuter VE, Gonen M, Kattan MW. A nomogram for predicting a positive repeat prostate biopsy in patients with a previous negative biopsy session. J Urol. 2003;170(4 Pt 1):1184-1188; discussion 1188.

23. Scattoni V, Roscigno M, Freschi M, et al. Atypical small acinar proliferation (ASAP) on extended prostatic biopsies: predictive factors of cancer detection on repeat biopsies. Arch Ital Urol Androl.2005;77(1):3136.

24. Chen R, Huang Y, Cai X, et al. Age-Specific Cutoff Value for the Application of Percent Free Prostate-Specific Antigen (PSA) in Chinese Men with Serum PSA Levels of 4.0-10.0 ng/ml. PLoS One.2015;10(6): $\mathrm{e} 0130308$.

\section{Hosted file}


Table.docx available at https://authorea.com/users/356083/articles/525384-a-new-asapscoring-system-and-risk-table-in-patients-with-atypical-small-acinar-proliferationasap-to-predict-the-second-prostate-biopsy-outcomes 